\title{
Effects of Attrition Shoes on Kinematics and Kinetics of Lower Limb Joints During Walking
}

\author{
Shane Fei Chen ${ }^{1}$, Yan Wang ${ }^{1,2}$, Yinghu Peng ${ }^{1}$ and Ming Zhang ${ }^{1,2 *}$ \\ ${ }^{1}$ Department of Biomedical Engineering, Faculty of Engineering, The Hong Kong Polytechnic University, Hong Kong, Hong Kong \\ SAR, China, ${ }^{2}$ Hong Kong Polytechnic University Shenzhen Research Institute, Shenzhen, China
}

Shoe attrition is inevitable as wearing time increases, which may produce diverse influences on kinematics and kinetics of lower limb joints. Excessive attrition may change support alignment and lead to deleterious impacts on the joints. The study identifies the biomechanical influences of aging shoes on lower limb joints. The shoes in the experiment were manually worn in the lateral heel. Nineteen healthy participants, including thirteen males and six females, were recruited to conduct walking experiments wearing attrition shoes (AS) and new shoes (NS) with a random order. A Vicon motion analysis system was used to collect kinematic data and ground reaction force. Kinematic and kinetic parameters of the hip, knee, and ankle joints were calculated using the

OPEN ACCESS

Edited by:

Lizhen Wang,

Beihang University, China

Reviewed by:

Qichang Mei,

Ningbo University, China Yaodong Gu,

Ningbo University, China

*Correspondence: Ming Zhang

ming.zhang@polyu.edu.hk

Specialty section:

This article was submitted to Biomechanics,

a section of the journal Frontiers in Bioengineering and Biotechnology

Received: 29 November 2021 Accepted: 17 January 2022 Published: 09 February 2022

Citation:

Chen SF, Wang $Y$, Peng $Y$ and Zhang M (2022) Effects of Attrition Shoes on Kinematics and Kinetics of Lower Limb Joints During Walking. Front. Bioeng. Biotechnol. 10:824297. doi: 10.3389/fbioe.2022.824297 Anybody Musculoskeletal Model and compared between the two conditions, AS and NS. The results showed that wearing an attrition shoe decreased the plantarflexion angle and plantarflexion moment of the ankle joint, while significantly increasing the magnitude of the first peak of the knee adduction moment and hip abduction moment and hip internal rotation moment $(p<.05)$. The results of the study implied that wearing attrition shoes is not recommended for those people with knee problems due to increase in medial loading.

Keywords: attrition shoes, lower extremity, multibody calculation, motion analysis, joint biomechanics

\section{INTRODUCTION}

Footwear is designed to protect humans from injuries in different environments (Barton et al., 2009) and provide assistance in motion control and attenuation of impact forces in daily activities. Shoes affect kinematics with a limitation of eversion and inversion range of motion of lower limb joints during walking (Morio et al., 2009; Sun et al., 2020; Dempster et al., 2021). However, aging attrition shoes (Moghaddam et al., 2019) change the plantar support surface and may affect the kinematics and kinetics of the lower limb, which might be related to joint injuries (Taunton et al., 2003; Ramsey et al., 2019). Compared with new shoes, worn shoes with decreasing cushioning capability lead to an increase of stance time and kinematic adaptational changes (Kong et al., 2009). Worn shoes were also demonstrated to increase energy cost and risk of injuries (Ramsey et al., 2018) and decrease lower limb stability (Saito et al., 2007). Footwear was found to reduce the external loading rate to protect lower limbs from injuries (Altman and Davis, 2016) and designed to reduce the knee adduction moment (Fisher et al., 2007; Peng et al., 2020) to relieve medial knee pain. Lateral-wedged insoles or shoes with a certain degree of inclination to the medial were normally adopted to be the interventions to decrease the first peak knee adduction moment that was beneficial to patients with medial knee osteoarthritis (Radzimski et al., 2012; Felson et al., 2019; Sinclair and Stainton, 2019). 
A

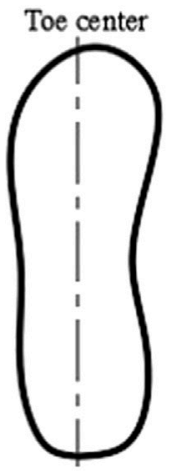

Heel center

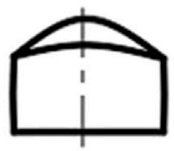

Schematic illustration of NS
B

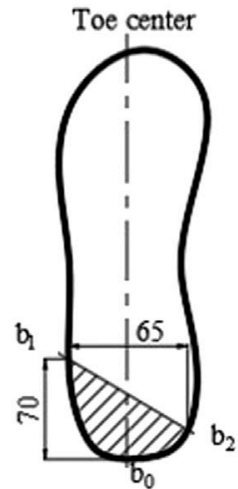

Heel center

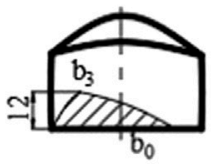

C

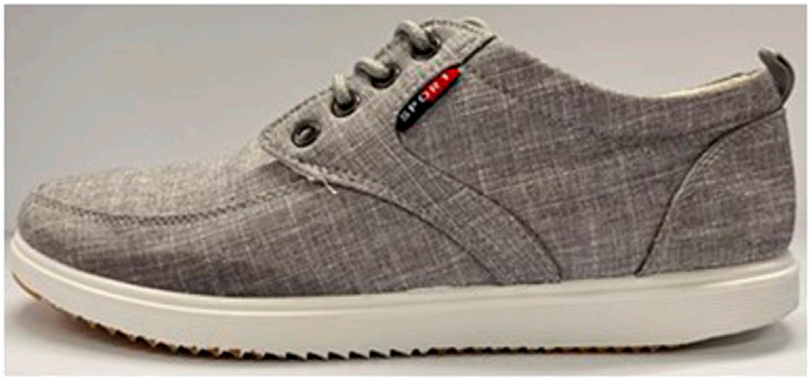

Prototype of AS

FIGURE 1 | Scheme of shoe conditions. (A) Schematic illustration of NS. (B) Schematic illustration of AS. (C) Prototype of AS.

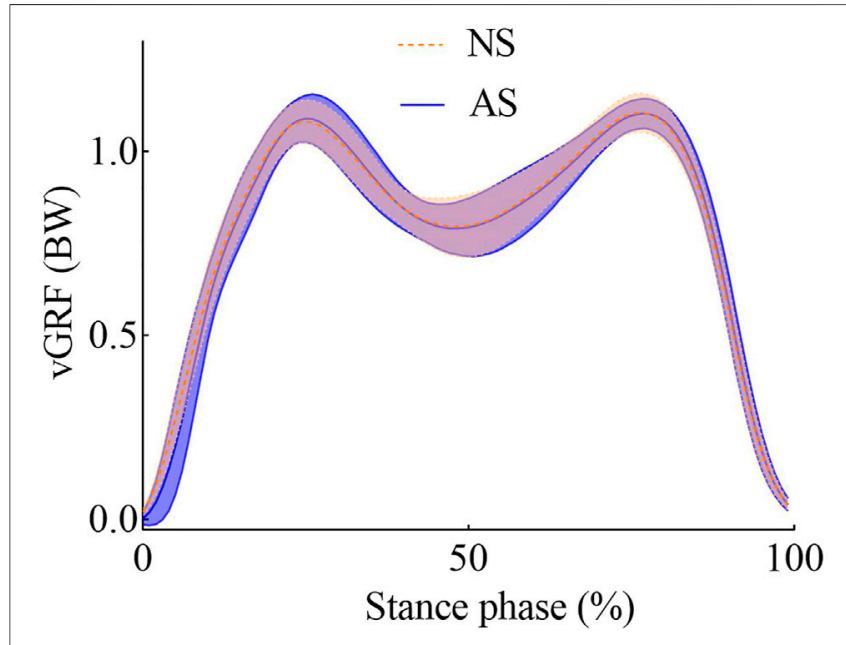

FIGURE 2 | Vertical GRF for NS and AS during stance.

Patterns of shoe attrition could be classified into lateral, medial, and central degradation (Baumfeld et al., 2015). Lateral heel degradation was found to be the most popular pattern according to a measurement of over 200 shoes, with an average shoe age of almost a year (Sole et al., 2014). In an assessment of 76 participants (Finestone et al., 2012), most of the outsole abrasion presented a posterolateral pattern with an average of $12^{\circ}$ and $5 \mathrm{~cm}^{3}$ abrasion volume. There is lack of studies to understand the biomechanical effects of shoe abrasion on lower limb biomechanics.

This study aimed to identify the immediate effects of worn shoes on kinematics and kinetics of the lower limb. As the most popular worn pattern, lateral heel attrition with mediolateral asymmetry was
TABLE 1 | Peak angles of lower limb joints for NS and AS during stance ( ${ }^{\circ}$.

\begin{tabular}{|c|c|c|c|c|}
\hline Parameter & NS mean (SD) & AS mean (SD) & $p$-values & $\mathbf{P}^{*}$ \\
\hline \multicolumn{5}{|l|}{ Hip } \\
\hline flexion & 26.199 (3.639) & 26.270 (3.672) & 0.761 & 0.027 \\
\hline extension & $-14.587(3.246)$ & $-14.611(3.509)$ & 0.923 & 0.041 \\
\hline external & $14.284(4.211)$ & $14.313(4.330)$ & 0.947 & 0.050 \\
\hline \multicolumn{5}{|l|}{ rotation } \\
\hline internal rotation & 4.708 (6.133) & 5.283 (6.782) & 0.141 & 0.014 \\
\hline abduction & 3.199 (2.468) & 3.235 (2.326) & 0.822 & 0.032 \\
\hline adduction & $-4.384(2.091)$ & $-4.372(1.861)$ & 0.946 & 0.045 \\
\hline \multicolumn{5}{|l|}{ Knee } \\
\hline flexion & 45.461 (5.358) & $45.357(4.665)$ & 0.864 & 0.036 \\
\hline \multicolumn{5}{|l|}{ Ankle } \\
\hline eversion & $-5.213(11.201)$ & $-6.053(10.003)$ & 0.230 & 0.018 \\
\hline inversion & $-23.019(11.700)$ & $-23.415(11.626)$ & 0.578 & 0.023 \\
\hline dorsiflexion & 24.499 (2.534) & 25.651 (3.339) & 0.057 & 0.009 \\
\hline plantarflexion & $-2.466(2.506)$ & $-0.828(3.336)$ & 0.027 & 0.005 \\
\hline
\end{tabular}

Significant difference at $\mathrm{p}$-value $<P^{*}$ when walking with AS was compared with walking with NS.

set as the experimental condition. It is hypothesized that lateral heel attrition would increase the knee adduction moments.

\section{METHODS}

\section{Participants}

Nineteen participants were recruited to participate in the study. This sample size was estimated using $G^{*}$ power 3.0.10, Universität 


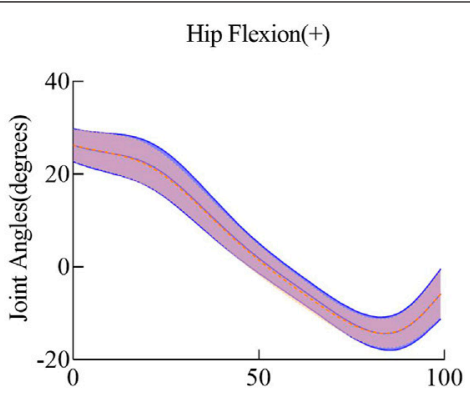

Hip Internal Rotation(+)

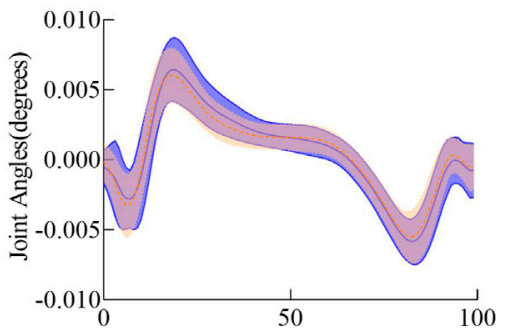

Ankle Dorsiflexion(+)

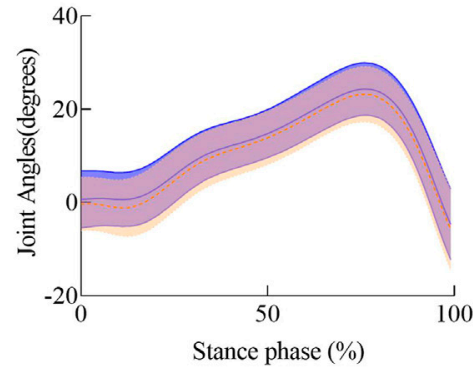

Hip Abduction $(+)$

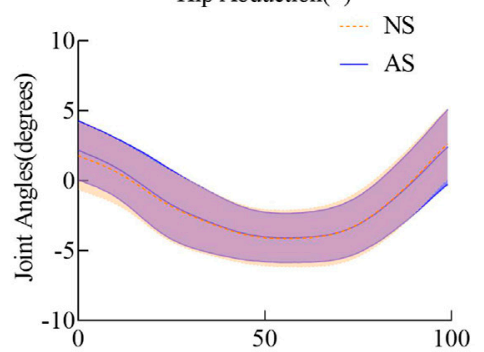

Knee Flexion(+)

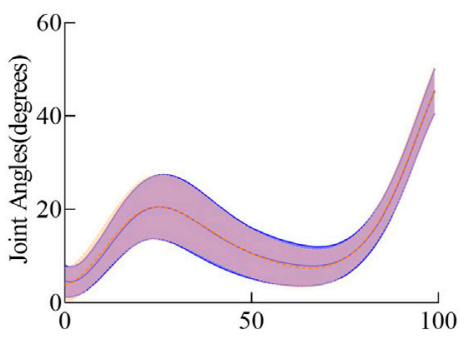

Ankle Eversion(+)

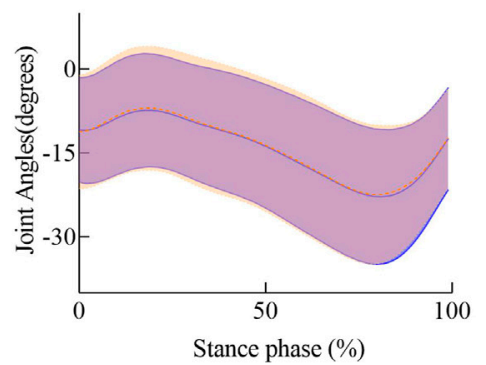

FIGURE 3 | Joint angles of lower extremities with shade \pm 1 std for NS and AS during the stance phase.

Düsseldorf, Germany, and the minimum sample size was 15 with a significant level of 0.05 and a statistical level of 0.8 . The medium effect size of 0.8 was adopted in the sample size calculation. The average age of participants was $25 \pm 5$ years. The means and standard deviations of body mass and height were $69.5 \pm 12.2 \mathrm{~kg}$ and $173.6 \pm 9.3 \mathrm{~cm}$, respectively. All participants had no histories of lower extremity injuries during the experiments. This study was approved by the Human Participants Ethics Sub-Committee of The Hong Kong Polytechnic University (Number: HSEARS20150121003). Informed consent was obtained from all participants before experiments.

\section{Footwear Conditions}

Canvas shoes were adopted for the preparation of non-attrition (new shoes, NS) and attrition shoes (attrition shoes, AS). Attrition shoes (AS) with lateral worn heels were adopted, which is the most popular attrition pattern (Finestone et al., 2012; Sole et al., 2014). As shown in Figure 1B, the degree of attrition was measured by the length of two lines, where one is the line connecting the lateral attrition point $b_{1}$ and heel center $b_{0}$ and the other is the line connecting the lateral attrition point $b_{1}$ and the other attrition point $b_{2}$. The maximum attrition position is at point $b_{3}$ in the lateral heel portion, as shown in Figure 1B. A prototype of AS is shown in Figure 1C.

\section{Experiment Setup and Procedure}

A Vicon motion capture system with eight cameras (Vicon, Oxford Metrics Ltd., Oxford, England) was used to collect motion data at $150 \mathrm{~Hz}$, and two force platforms (OR6, AMTI, Watertown, United States) were used to measure the ground reaction force $(\mathrm{GRF})$ at $1000 \mathrm{~Hz}$. The participants wore tightfitting clothes, and reflective markers were attached on bony landmarks to define the lower limb into seven segments. The marker set followed a previous study (Peng et al., 2020). The participants were required to walk for a few minutes to accommodate each pair of shoes in random order. They were required to walk at their natural walking speed during the walking trials. Each participant completed three successful trials with each condition of shoes. 
TABLE 2 | Peak moments of lower limb joints for NS and AS during stance (BW and. $\mathrm{BH})$.

\begin{tabular}{|c|c|c|c|c|}
\hline Parameter & NS mean (SD) & AS mean (SD) & $p$-value & $\mathbf{P}^{*}$ \\
\hline \multicolumn{5}{|l|}{ Hip } \\
\hline $\begin{array}{l}\text { flexion rotation } \\
\text { extension rotation } \\
\text { external rotation } \\
\text { internal rotation } \\
\text { first abduction } \\
\text { second abduction }\end{array}$ & $\begin{array}{r}0.044(0.012) \\
-0.035(0.012) \\
0.006(0.002) \\
-0.005(0.002) \\
0.046(0.008) \\
0.043(0.008)\end{array}$ & $\begin{array}{r}0.043(0.012) \\
-0.038(0.014) \\
0.007(0.002) \\
-0.006(0.002) \\
0.047(0.008) \\
0.045(0.008)\end{array}$ & $\begin{array}{c}0.443 \\
0.130 \\
0.062 \\
0.000^{1} \\
0.049 \\
0.004^{1}\end{array}$ & $\begin{array}{l}0.039 \\
0.022 \\
0.017 \\
0.003 \\
0.014 \\
0.006\end{array}$ \\
\hline \multicolumn{5}{|l|}{ Knee } \\
\hline $\begin{array}{l}\text { Flexion } \\
\text { extension } \\
\text { external rotation } \\
\text { internal rotation } \\
\text { first adduction } \\
\text { second adduction }\end{array}$ & $\begin{array}{r}0.033(0.016) \\
-0.015(0.009) \\
0.001(0.001) \\
-0.002(0.001) \\
-0.024(0.004) \\
-0.020(0.006)\end{array}$ & $\begin{array}{r}0.034(0.015) \\
-0.014(0.009) \\
0.001(0.001) \\
-0.002(0.001) \\
-0.026(0.004) \\
-0.021(0.005)\end{array}$ & $\begin{array}{c}0.286 \\
0.064 \\
0.982 \\
0.186 \\
0.006^{1} \\
0.310\end{array}$ & $\begin{array}{l}0.033 \\
0.019 \\
0.050 \\
0.028 \\
0.008 \\
0.036\end{array}$ \\
\hline \multicolumn{5}{|l|}{ Ankle } \\
\hline $\begin{array}{l}\text { eversion } \\
\text { inversion } \\
\text { external rotation } \\
\text { internal rotation } \\
\text { dorsiflexion } \\
\text { plantarflexion }\end{array}$ & $\begin{array}{r}0.018(0.007) \\
-0.005(0.001) \\
0.003(0.001) \\
-0.008(0.002) \\
0.090(0.008) \\
-0.008(0.002)\end{array}$ & $\begin{array}{r}0.019(0.008) \\
-0.005(0.001) \\
0.003(0.001) \\
-0.008(0.002) \\
0.089(0.007) \\
-0.007(0.003)\end{array}$ & $\begin{array}{l}0.173 \\
0.816 \\
0.452 \\
0.926 \\
0.233 \\
0.011^{1}\end{array}$ & $\begin{array}{l}0.025 \\
0.044 \\
0.042 \\
0.047 \\
0.031 \\
0.011\end{array}$ \\
\hline
\end{tabular}

${ }^{1}$ Significant difference at $\mathrm{p}$-value $<P^{*}$ when walking with AS was compared with walking with NS.

\section{Musculoskeletal Model}

The lower limb musculoskeletal (MSK) model using the Anybody Modeling System (version 6.0.3, AnyBody Technology, Aalborg, Denmark) was used in this study. The generic MSK model was based on the anthropometric database of the Twente Lower Extremity Model (TLEM 1.1) (Klein Horsman et al., 2007). The experimental data provided input for the MSK model to calculate the kinematics and kinetics of the lower extremity joints (Peng et al., 2018). The marker trajectory data and anthropometrics for each participant were used to optimize the MSK model, and then, kinematic parameters of the lower limb joints were calculated. After the kinematical calculation, inverse dynamic calculation was conducted to obtain kinetics of the joints.

\section{Statistical Analysis}

SPSS (Version 22.0, IBM, Chicago, IL, United States) was used to perform statistical analysis. Peak values of angles and moments of ankle, knee, and pelvis joints and vertical GRF were extracted for statistical analysis of the two conditions. The joint moments were normalized with body weight and body height (BW and·BH) (Peng et al., 2020). Mean values of all variables were calculated from three successful trials of each shoe condition for each participant. The data distribution was testified to be normal when the Shapiro-Wilk test with significance level 0.05 was performed. Post hoc paired t-tests with Benjamini-Hochberg adjustment $\left(\mathrm{P}^{\star}\right)$ were conducted to determine the relationship between the two conditions with a significance level of 0.05 .

\section{RESULTS}

Figure 2. shows the mean vertical GRF during the stance phase. The vertical GRFs were normalized by the body weight. Mean vertical GRF for different shoe conditions was calculated and showed no statistical difference with $p>.05$.

The mean and standard deviation (SD) of peak angles of lower extremity joints for the two conditions for all participants are listed in Table 1. Joint angles of lower extremities showed no significant difference during the stance phase shown in Figure 3.

The joint moments were normalized by dividing the body weight and body height (BW and $\mathrm{BH}$ ) of each participant. Details of joint moments are shown in Table 2. The amplitudes of the hip internal rotation moment and the second hip abduction moment increased significantly in the AS group. The first peak of the knee adduction moment for the AS group revealed an increase of $8.3 \%$ compared with that of the NS group. The peak moments of the ankle joint showed a slight decrease. Joint moments during the stance phase are shown in Figure 4.

\section{DISCUSSION}

In this study, the effects of attrition shoes on lower limb joints were revealed by comparing them with those of new shoes during walking. Joint angles showed no significant effects, but some of joint moments of the lower limb displayed significant changes. The amplitudes of the ankle plantarflexion moment decrease significantly in the AS group as compared to those of the NS group. However, the amplitudes of the second hip abduction moment and internal rotation moment increased significantly when participants wore attrition shoes. Meanwhile, the first peak of the knee adduction moment revealed a significant increase of $8.3 \%$ when the AS group was compared with the NS group.

For the ankle joint, though the amplitude of the plantarflexion angle showed no significant effects, it tends to decrease while walking in worn shoes. Meanwhile, the amplitude of the plantarflexion moment showed a slightly significant decrease. AS lack one wedge part in the lateral heel, which prolongs the onset of heel strike. Thus, it leads to a shortening time for the plantarflexion. The duration of the plantarflexion is decreased, which makes the foot dorsiflexion quick. However, during the loading phase, the plantarflexion of the ankle joint provides eccentric contraction for the tibialis anterior muscle (Jafarnezhadgero et al., 2020). The reduction of the plantarflexion would cause the tibialis anterior muscle to provide less contraction force, which results in larger landing impact. The lateral heel attrition shifts the contact center anteriorly, which reduces the lever arm that lowers. The reduction of the lever led to decrease of the peak of the plantarflexion moment as shown in Figure 4. Insufficient plantarflexion moment may generate a more flattening longitudinal arch (Kirby, 2017), which would lead to an increase of tensional force in the plantar fascia that has been reported to be one of the causes of ankle pain (Kogler et al., 

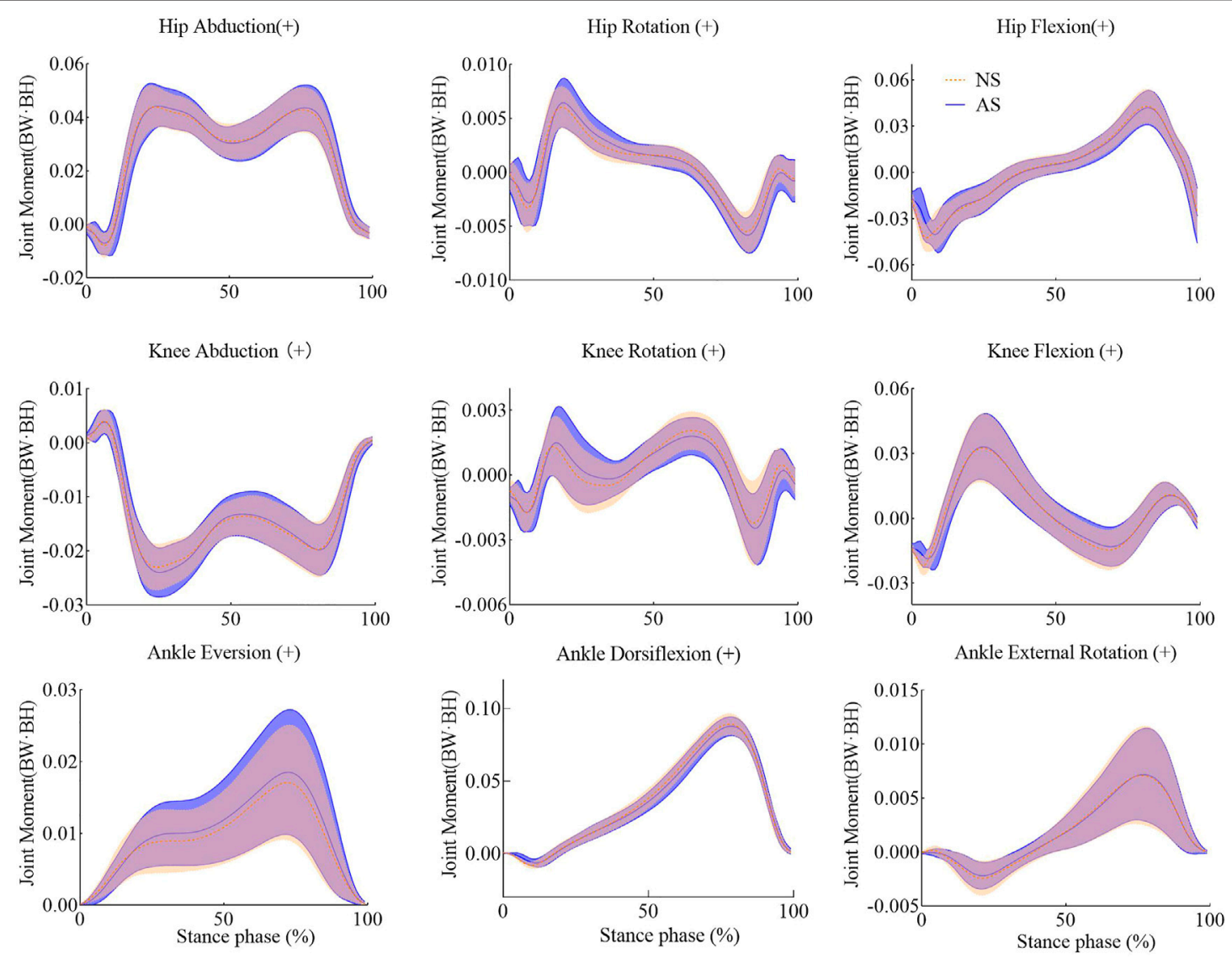

FIGURE 4 | Joint moments of lower extremities with shade \pm 1 std for NS and AS during the stance phase.

1996). Furthermore, the plantarflexion moment plays an important role in counterbalance (Kirby, 2017). Previous studies have indicated that plantarflexors regulate wholebody angular momentum in the sagittal plane during balance control (Neptune and Mcgowan, 2011), and fallers created a decreased plantarflexion moment compared to non-fallers (Barak et al., 2006). Consequently, the lack of plantarflexion moment in the AS group may also cause problems in balance.

Kinematic parameters of knee joints show no significant differences between the NS group and AS group. However, the first peak of the knee adduction moment increases by $8.3 \%$ with a significant level of $p=.006$, as shown in Table 2 and Figure 4. Since the attrition concentrates on the lateral heel, there is no significant effect in the second peak of the knee adduction moment. Wedge soles are often adopted as interventions for knee osteoarthritis (OA) (Desmyttere et al., 2018). Although the attrition outsole in this study is not exactly like wedge outsoles, the heel of AS displays the same appearance with medial wedges to a certain degree. The increase of the amplitude of the knee adduction moment agrees with that found in the previous studies (Lewinson et al., 2013; Peng et al., 2020), which illustrates that the peak knee adduction moment increases as the wedge develops from lateral to medial. On the contrary, lateral wedges are beneficial for participants with medial knee OA patients because they can reduce the peak of the knee adduction moment; the AS is disagreeable for them as it increases the knee adduction moment. Higher knee adduction moment has also been reported to have a relationship with the development of knee pain (Mai and Willwacher, 2019). Consequently, the aging shoes are also not suitable for participants with knee pain.

Aging shoes make an impact on the kinetics of the hip joint. For the AS group, the peak moments of the hip internal rotation and abduction increase significantly when compared with the NS group as illustrated in Table 2 and Figure 4. Aging shoes were found to lower activities of the vastus medialis and rectus femoris muscles and strengthen the activity of the gluteus medius muscle during the loading phase while walking (Jafarnezhadgero et al., 2020). The activities of these muscles may lead to increase of hip internal rotation and abduction moments. Furthermore, the increase of the hip internal rotation moment may cause abnormal hip motions (Weidow et al., 2006), which may change the rotational alignment of other segments in the lower limb.

A few limitations in the study should be discussed. The present study examines the immediate effect of shoe degradation on 
lower limb joints. However, the wear of shoes was a persistent behavior, of which impact is gradual. Canvas shoes with flat outsoles were adopted in this study, but other types of shoes were not explored. The lateral heel of shoes was worn mechanically, which may have slight differences with naturally worn shoes. The sample size in the study was another limitation. The generalizability of the findings should be noted. Additionally, further research should identify the gender difference in shoe attrition. The effects of other attrition patterns on the lower limb joints require further research.

\section{CONCLUSION}

Lateral heel attrition in outsoles is the most common degeneration pattern of shoes. The attrition shoes have significant impacts on the kinematics and kinetics of the lower limb joints. The peak plantarflexion angle and moment of the ankle joint decrease, and the first knee adduction moment and hip internal and abduction moments increase during walking for the AS group. Our findings in this study imply that aging shoes are not desirable, especially for those people with knee problems. Attrition in the heel also raises balance risk. Although the exact abrasion amount in aging shoes is not demonstrated for a shoe change, attrition shoes influence the walking pattern and result in discomfort.

\section{REFERENCES}

Altman, A. R., and Davis, I. S. (2016). Prospective Comparison of Running Injuries between Shod and Barefoot Runners. Br. J. Sports Med. 50, 476-480. doi:10. 1136/bjsports-2014-094482

Barak, Y., Wagenaar, R. C., and Holt, K. G. (2006). Gait Characteristics of Elderly People with a History of Falls: a Dynamic Approach. Phys. Ther. 86, 1501-1510. doi:10.2522/ptj.20050387

Barton, C. J., Bonanno, D., and Menz, H. B. (2009). Development and Evaluation of a Tool for the Assessment of Footwear Characteristics. J. Foot Ankle Res. 2, 1-12. doi:10.1186/1757-1146-2-10

Baumfeld, D., Raduan, F. C., Macedo, B., Silva, T. A. A., Baumfeld, T., Favato, D. F., et al. (2015). Shoe Heel Abrasion and its Possible Biomechanical Cause: a Transversal Study with Infantry Recruits. J. Orthop. Surg. Res. 10, 1-5. doi:10. 1186/s13018-015-0319-0

Dempster, J., Dutheil, F., and Ugbolue, U. C. (2021). The Prevalence of Lower Extremity Injuries in Running and Associated Risk Factors: A Systematic Review. Phys. Activity Health 5, 133-145. doi:10.5334/paah.109

Desmyttere, G., Hajizadeh, M., Bleau, J., and Begon, M. (2018). Effect of Foot Orthosis Design on Lower Limb Joint Kinematics and Kinetics during Walking in Flexible Pes Planovalgus: A Systematic Review and Meta-Analysis. Clin. Biomech. 59, 117-129. doi:10.1016/j.clinbiomech.2018.09.018

Felson, D. T., Parkes, M., Carter, S., Liu, A., Callaghan, M. J., Hodgson, R., et al. (2019). The Efficacy of a Lateral Wedge Insole for Painful Medial Knee Osteoarthritis after Prescreening: A Randomized Clinical Trial. Arthritis Rheumatol. 71, 908-915. doi:10.1002/art.40808

Finestone, A. S., Petrov, K., Agar, G., Honig, A., Tamir, E., and Milgrom, C. (2012). Pattern of Outsole Shoe Heel Wear in Infantry Recruits. J. Foot Ankle Res. 5, 1-7. doi:10.1186/1757-1146-5-27

Fisher, D. S., Dyrby, C. O., Mündermann, A., Morag, E., and Andriacchi, T. P. (2007). In Healthy Subjects without Knee Osteoarthritis, the Peak Knee Adduction Moment Influences the Acute Effect of Shoe Interventions Designed to Reduce Medial Compartment Knee Load. J. Orthop. Res. 25, 540-546. doi:10.1002/jor.20157

\section{DATA AVAILABILITY STATEMENT}

The raw data supporting the conclusion of this article will be made available by the authors, without undue reservation.

\section{ETHICS STATEMENT}

The studies involving human participants were reviewed and approved by the Human Participants Ethics Sub-Committee of The Hong Kong Polytechnic University (Number: HSEARS20150121003). The patients/participants provided their written informed consent to participate in this study.

\section{AUTHOR CONTRIBUTIONS}

SC: project conception, manuscript draft, and revising. YW: manuscript revising. YP: project conception and manuscript draft. MZ: manuscript revising and supervision.

\section{FUNDING}

This study was supported by the National Science Foundation of China (NSFC) (Numbers: 11732015 and 11972315).

Jafarnezhadgero, A. A., Anvari, M., and Granacher, U. (2020). Long-term Effects of Shoe Mileage on Ground Reaction Forces and Lower Limb Muscle Activities during Walking in Individuals with Genu Varus. Clin. Biomech. 73, 55-62. doi:10.1016/j.clinbiomech.2020.01.006

Kirby, K. A. (2017). Longitudinal Arch Load-Sharing System of the Foot. Revista Española de Podología 28, e18-e26. https://docs.vicon.com/pages/viewpage. action?pageId=50888852. doi:10.1016/j.repod.2017.03.003

Klein Horsman, M. D., Koopman, H. F. J. M., Van Der Helm, F. C. T., Prosé, L. P., and Veeger, H. E. J. (2007). Morphological Muscle and Joint Parameters for Musculoskeletal Modelling of the Lower Extremity. Clin. Biomech. 22, 239-247. doi:10.1016/j.clinbiomech.2006.10.003

Kogler, G., Solomonidis, S., and Paul, J. (1996). Biomechanics of Longitudinal Arch Support Mechanisms in Foot Orthoses and Their Effect on Plantar Aponeurosis Strain. Clin. Biomech. 11, 243-252. doi:10.1016/02680033(96)00019-8

Kong, P. W., Candelaria, N. G., and Smith, D. R. (2009). Running in New and Worn Shoes: a Comparison of Three Types of Cushioning Footwear. Br. J. Sports Med. 43, 745-749. doi:10.1136/bjsm.2008.047761

Lewinson, R. T., Fukuchi, C. A., Worobets, J. T., and Stefanyshyn, D. J. (2013). The Effects of Wedged Footwear on Lower Limb Frontal Plane Biomechanics during Running. Clin. J. Sport Med. 23, 208-215. doi:10. 1097/jsm.0b013e31826b7c83

Mai, P., and Willwacher, S. (2019). Effects of Low-Pass Filter Combinations on Lower Extremity Joint Moments in Distance Running. J. Biomech. 95, 109311. doi:10.1016/j.jbiomech.2019.08.005

Moghaddam, S. R. M., Hemler, S. L., Redfern, M. S., Jacobs, T. D. B., and Beschorner, K. E. (2019). Computational Model of Shoe Wear Progression: Comparison with Experimental Results. Wear 422-423, 235-241. doi:10.1016/j. wear.2019.01.070

Morio, C., Lake, M. J., Gueguen, N., Rao, G., and Baly, L. (2009). The Influence of Footwear on Foot Motion during Walking and Running. J. Biomech. 42, 2081-2088. doi:10.1016/j.jbiomech.2009.06.015

Neptune, R. R., and Mcgowan, C. P. (2011). Muscle Contributions to Whole-Body Sagittal Plane Angular Momentum during Walking. J. Biomech. 44, 6-12. doi:10.1016/j.jbiomech.2010.08.015 
Peng, Y., Wong, D. W.-C., Wang, Y., Chen, T. L.-W., Tan, Q., Chen, Z., et al. (2020). Immediate Effects of Medially Posted Insoles on Lower Limb Joint Contact Forces in Adult Acquired Flatfoot: A Pilot Study. Ijerph 17, 2226. doi:10.3390/ijerph17072226

Peng, Y., Zhang, Z., Gao, Y., Chen, Z., Xin, H., Zhang, Q., et al. (2018). Concurrent Prediction of Ground Reaction Forces and Moments and Tibiofemoral Contact Forces during Walking Using Musculoskeletal Modelling. Med. Eng. Phys. 52, 31-40. doi:10.1016/j.medengphy.2017.11.008

Radzimski, A. O., Mündermann, A., and Sole, G. (2012). Effect of Footwear on the External Knee Adduction Moment - A Systematic Review. The Knee 19, 163-175. doi:10.1016/j.knee.2011.05.013

Ramsey, C. A., Cury Ribeiro, D., Lamb, P., Sole, C. C., and Sole, G. (2018). Reliability of the Footwear Total Asymmetry Score Tool. Footwear Sci. 10, 119-128. doi:10.1080/19424280.2018.1478888

Ramsey, C. A., Lamb, P., Kaur, M., Baxter, G. D., and Ribeiro, D. C. (2019). "How Are Running Shoes Assessed? A Systematic Review of Characteristics and Measurement Tools Used to Describe Running Footwear". J. Sports Sci. 37, 1617-1629. doi:10.1080/02640414.2019.1578449

Saito, S., Muraki, S., and Tochihara, Y. (2007). Effects of Worn-Out Soles on Lower Limb Stability, Shock Absorption and Energy Cost during Prolonged Walking. J. Physiol. Anthropol. 26, 521-526. doi:10.2114/jpa2.26.521

Sinclair, J., and Stainton, P. (2019). Effects of Medial and Lateral Wedged Orthoses on Knee and Ankle Joint Loading in Female Runners. Kinesiology (Zagreb, Online) 51, 189-197. doi:10.26582/k.51.2.9

Sole, C. C., Milosavljevic, S., Sole, G., and Sullivan, S. J. (2014). Patterns of Mediolateral Asymmetry in Worn Footwear. Footwear Sci. 6, 177-192. doi:10.1080/19424280.2014.913694
Sun, D., Fekete, G., Baker, J. S., and Gu, Y. (2020). Foot Motion Character during Forward and Backward Walking with Shoes and Barefoot. J. Mot. Behav. 52, 214-225. doi:10.1080/00222895.2019.1605972

Taunton, J. E., Ryan, M. B., Clement, D. B., Mckenzie, D. C., Lloyd-Smith, D. R., and Zumbo, B. D. (2003). A Prospective Study of Running Injuries: the Vancouver Sun Run "In Training" Clinics. Br. J. Sports Med. 37, 239-244. doi:10.1136/bjsm.37.3.239

Weidow, J., Tranberg, R., Saari, T., and Kärrholm, J. (2006). Hip and Knee Joint Rotations Differ between Patients with Medial and Lateral Knee Osteoarthritis: Gait Analysis of 30 Patients and 15 Controls. J. Orthop. Res. 24, 1890-1899. doi:10.1002/jor.20194

Conflict of Interest: The authors declare that the research was conducted in the absence of any commercial or financial relationships that could be construed as a potential conflict of interest.

Publisher's Note: All claims expressed in this article are solely those of the authors and do not necessarily represent those of their affiliated organizations, or those of the publisher, the editors, and the reviewers. Any product that may be evaluated in this article, or claim that may be made by its manufacturer, is not guaranteed or endorsed by the publisher.

Copyright (c) 2022 Chen, Wang, Peng and Zhang. This is an open-access article distributed under the terms of the Creative Commons Attribution License (CC BY). The use, distribution or reproduction in other forums is permitted, provided the original author(s) and the copyright owner(s) are credited and that the original publication in this journal is cited, in accordance with accepted academic practice. No use, distribution or reproduction is permitted which does not comply with these terms. 